PROCEEDINGS OF THE

AMERICAN MATHEMATICAL SOCIETY

Volume 127, Number 12, Pages 3439-3444

S 0002-9939(99)05008-X

Article electronically published on July 20, 1999

\title{
INVARIANT MEASURES FOR CERTAIN LINEAR FRACTIONAL TRANSFORMATIONS MOD 1
}

\author{
KARLHEINZ GRÖCHENIG AND ANDREW HAAS
}

(Communicated by Linda Keen)

\begin{abstract}
Explicit invariant measures are derived for a family of finite-toone, ergodic transformations of the unit interval having indifferent periodic orbits.
\end{abstract}

Examples of interesting, non-trivial maps of $[0,1]$ for which one can readily compute an invariant measure absolutely continuous to Lebesgue measure are not easy to come by. The familiar examples are the Gauss map, the backward continued fraction map, and other very special cases which are close in form to the first two. See [1], [3], and [4] for an overview of the literature. Maps for which the invariant measure is infinite are even less in evidence. We will consider a family of mappings $T_{k, n}$ of the unit interval that are essentially finite-to-one analogues of the backward continued fraction maps $T_{k}=\left\langle\frac{1}{u_{k}(1-x)}\right\rangle$ studied in [4], where $u_{k}=4 \cos ^{2} \frac{\pi}{k+2}$ and $\langle x\rangle$ is the fractional part of $x$. Both $T_{k}$ and $T_{k, n}$ are Möbius transformations mod 1 having indifferent periodic orbits of period $k$ containing zero. Surprisingly, for fixed $k, T_{k, n}$ converges uniformly to $T_{k}$ on compact subsets of $[0,1)$.

An explicit formula will be given for a $T_{k, n}$-invariant measure that is absolutely continuous to Lebesgue measure on $[0,1]$. The measure is infinite and the density $\rho_{k, n}$ is $C^{\infty}$ in the complement of the indifferent periodic orbit. Also, $\rho_{k, n}$ converges to the $T_{k}$-invariant density $\rho_{k}$ derived in [4].

Using Thaler's analysis in [6] of mappings of $[0,1]$ with indifferent fixed points, it is possible to show that the maps $T_{k, n}$ are ergodic with respect to Lebesgue measure.

From now on suppose that $k>0$ and $n>1$ in $\mathbb{N}$ have been fixed and that if $k=1$, then $n>2$. We begin by defining the Möbius transformation that will determine $T_{k, n}$. Write

$$
A_{\alpha}(x)=\frac{-\alpha x-n \alpha+\alpha}{(n-\alpha-1) x-n+1} .
$$

Then $A_{\alpha}(0)=\alpha, A_{\alpha}(1)=n$, and $A_{\alpha}^{-1}(x)=\frac{(n-1) x-n \alpha+\alpha}{(n-\alpha-1) x+\alpha} . A_{\alpha}$ has the following properties.

Received by the editors January 1, 1998.

1991 Mathematics Subject Classification. Primary 11J70, 58F11, 58F03.

Key words and phrases. Continued fractions, interval maps, invariant measures.

The second author would like to thank the University of Washington for kindly providing access to their research facilities while this paper was in preparation.

(C)1999 American Mathematical Society 
Proposition 1. For every integer $n \geq 2$ there exists a sequence $\alpha_{k}$ depending on $n, \alpha_{1}=1>\alpha_{2}=1 / 2>\alpha_{3}>\cdots>\alpha^{*}=\frac{n-1}{4 n-3}$, such that $A_{\alpha_{k}}^{k}(0)=1$ and for each $\alpha^{*} \leq \alpha \leq \alpha_{k}, 0<A_{\alpha}(0)<A_{\alpha}^{2}(0)<\cdots<A_{\alpha}^{k}(0) \leq 1$, and $A_{\alpha}^{k}(0)$ is an increasing function in $\alpha$.

Proof. We argue by induction. Since $0<A_{\alpha}(0)=\alpha \leq 1$, for $0<\alpha \leq 1$, the proposition is true for $k=1$. Suppose that it is true for a $k>1$.

When $\alpha^{*}<\alpha<n-1$, then $A_{\alpha}$ has no real fixed point, and by direct computation,

$$
x<A_{\alpha}(x) \quad \text { if } x<A_{\alpha}^{-1}(\infty)=\frac{n-1}{n-\alpha-1} .
$$

$A_{\alpha^{*}}$ is a parabolic Möbius transformation with a unique fixed point at $x=1 / 2$. The $A_{\alpha^{*}}$-orbit of 0 looks like $0<A_{\alpha^{*}}(0)<A_{\alpha^{*}}^{2}(0)<\cdots<1 / 2$.

Consider the function $f_{k}(\alpha)=A_{\alpha}^{k}(0)-A_{\alpha}^{-1}(1)=A_{\alpha}^{k}(0)-1+\alpha . f_{k}$ is continuous and increasing on $\left[\alpha^{*}, \alpha_{k}\right]$ and $f_{k}\left(\alpha^{*}\right)<\alpha^{*}-1 / 2<0<f_{k}\left(\alpha_{k}\right)=\alpha_{k}$ by induction. Consequently, there is a value $a_{k+1} \in\left(\alpha^{*}, \alpha_{k}\right)$ with $f_{k}\left(\alpha_{k+1}\right)=0$, or $A_{\alpha_{k+1}}^{k+1}(0)=1$.

By (1) and the induction hypothesis, since $\alpha_{k+1}<\alpha_{k}$,

$$
0<A_{\alpha_{k+1}}(0)<A_{\alpha_{k+1}}^{2}(0)<\cdots<A_{\alpha_{k+1}}^{k}(0)<1=A_{\alpha_{k+1}}^{k+1}(0) .
$$

Furthermore, since both $\frac{\partial}{\partial x} A_{\alpha}(x)>0$ and $\frac{\partial}{\partial \alpha} A_{\alpha}(x)>0$ for $0 \leq x<1$ and $0<\alpha \leq 1$, the chain rule and the induction hypothesis yield

$$
\frac{\partial}{\partial \alpha} A_{\alpha}^{k+1}(x)=\frac{\partial A_{\alpha}}{\partial \alpha}\left(A_{\alpha}^{k}(x)\right)+\frac{\partial A_{\alpha}}{\partial x}\left(A_{\alpha}^{k}(x)\right) \cdot \frac{\partial A_{\alpha}^{k}}{\partial \alpha}(x)>0
$$

if $A_{\alpha}^{k}(x)<1$. Consequently $A_{\alpha}^{k+1}(0)<1$ for $\alpha<\alpha_{k+1}$.

From now on we fix $k \geq 1$ and write $A_{\alpha_{k}}=A$.

Proposition 2. $\left(A^{k}\right)^{\prime}(0)=1$.

This proposition is equivalent to the statement that $T_{k, n}$ is an indifferent periodic orbit of period $k$; that is the orbit of 0 . To prove the proposition, we need a simple, useful identity.

Lemma 1. For any Möbius transformation $C$ and $z, w \in \mathbb{C}$ with $C(z), C(w) \neq \infty$ we have

$$
\left(\frac{C(z)-C(w)}{z-w}\right)^{2}=C^{\prime}(z) C^{\prime}(w)
$$

Proof. Without loss of generality $C(z)=\frac{a z+b}{c z+d}$ with $a d-b c=1$. Then

$$
\begin{aligned}
& (C(z)-C(w))^{2}=\left(\frac{a z+b}{c z+d}-\frac{a w+b}{c w+d}\right)^{2} \\
= & \left(\frac{(a z+b)(c w+d)-(a w+b)(c z+d)}{(c z+d)(c w+d)}\right)^{2} \\
= & \frac{(a d-b c)^{2}(z-w)^{2}}{(c z+d)^{2}(c w+d)^{2}}=(z-w)^{2} C^{\prime}(z) C^{\prime}(w) .
\end{aligned}
$$




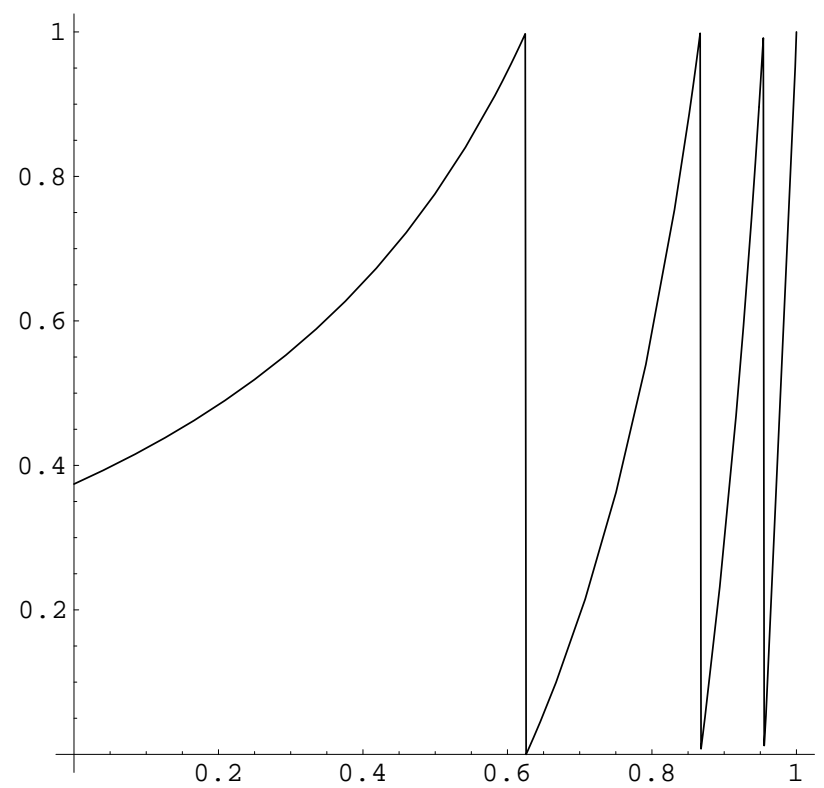

FiguRE 1

Proof of Proposition 2. From Lemma 1 we have

$$
A^{\prime}\left(A^{i}(0)\right) A^{\prime}\left(A^{i-1}(0)\right)=\left(\frac{A^{i+1}(0)-A^{i}(0)}{A^{i}(0)-A^{i-1}(0)}\right)^{2} .
$$

Therefore

$$
\begin{aligned}
{\left[\left(A^{k}\right)^{\prime}(0)\right]^{2} } & =\left[\prod_{i=0}^{k-1} A^{\prime}\left(A^{i}(0)\right)\right]^{2} \\
& =A^{\prime}(0) \cdot A^{\prime}\left(A^{k-1}(0)\right) \cdot \prod_{i=1}^{k-1}\left(A^{\prime}\left(A^{i}(0)\right) A^{\prime}\left(A^{i-1}(0)\right)\right) \\
& =A^{\prime}(0) \cdot A^{\prime}\left(A^{k-1}(0)\right) \cdot \prod_{i=1}^{k-1}\left(\frac{A^{i+1}(0)-A^{i}(0)}{A^{i}(0)-A^{i-1}(0)}\right)^{2} \\
& =A^{\prime}(0) \cdot A^{\prime}\left(A^{k-1}(0)\right)\left(\frac{1-A^{k-1}(0)}{A(0)}\right)^{2} .
\end{aligned}
$$

Using $A^{k}(0)=1$, we can write $A^{k-1}(0)=A^{-1}(1)$ and $A^{\prime}\left(A^{k-1}(0)\right)=\frac{1}{\left(A^{-1}\right)^{\prime}(1)}$. Then we continue with

$$
\left[\left(A^{k}\right)^{\prime}(0)\right]^{2}=\frac{A^{\prime}(0)\left(1-A^{-1}(1)\right)^{2}}{\left(A^{-1}\right)^{\prime}(1)(A(0))^{2}}=\frac{\frac{\alpha(n-\alpha)}{n-1} \cdot \alpha^{2}}{\frac{\alpha(n-\alpha)}{n-1} \cdot \alpha^{2}}=1 .
$$

Define $T(x)=\langle A(x)\rangle$. See Figure 1 for the graph of $T$, when $k=3$ and $n=4$. 
Theorem 1. The function

$$
\rho(x)=\left(\sum_{i=0}^{k-1} \frac{1}{x-A^{i}(0)} \chi_{\left[A^{i}(0), A^{i+1}(0)\right)}(x)\right)-\frac{1}{x+n-1}
$$

is an invariant density for $T$.

Proof. The density $\rho$ of an absolutely continuous $T$-invariant measure is an eigenfunction of eigenvalue 1 of the Perron-Frobenius operator [5]

$$
L_{T} \rho(x)=\sum_{\{y \mid T y=x\}} \frac{1}{\left|T^{\prime}(y)\right|} \rho(y) .
$$

First suppose that $x \in\left[A^{i}(0), A^{i+1}(0)\right)$ for $i>0$. Then $x$ has two types of preimages under $T$ : one in $\left[A^{i-1}(0), A^{i}(0)\right)$ and $n-1$ others in $\left[A^{-1}(1), 1\right)$.

$$
\begin{aligned}
L_{T} \rho(x)= & \frac{1}{A^{\prime}\left(A^{-1}(x)\right)}\left(\frac{1}{A^{-1}(x)-A^{i-1}(0)}-\frac{1}{A^{-1}(x)+n-1}\right) \\
& +\sum_{j=1}^{n-1} \frac{1}{A^{\prime}\left(A^{-1}(x+j)\right)}\left(\frac{1}{A^{-1}(x+j)-A^{-1}(1)}-\frac{1}{A^{-1}(x+j)+n-1}\right) \\
= & \mathcal{U}+\mathcal{V}
\end{aligned}
$$

where

$$
\mathcal{U}=\left(A^{-1}\right)^{\prime}(x)\left(\frac{n-1+A^{i-1}(0)}{\left(A^{-1}(x)-A^{i-1}(0)\right)\left(A^{-1}(x)+n-1\right)}\right)
$$

and

$$
\mathcal{V}=\sum_{j=1}^{n-1}\left(A^{-1}\right)^{\prime}(x+j)\left(\frac{A^{-1}(1)+n-1}{\left(A^{-1}(x+j)-A^{-1}(1)\right)\left(A^{-1}(x+j)+n-1\right)}\right) .
$$

First we simplify $\mathcal{U}$. Using Lemma 1 and $A(-n+1)=0$

$$
A^{-1}(x)-A^{i-1}(0)=\left(x-A^{i}(0)\right)\left(\left(A^{-1}\right)^{\prime}(x) \cdot\left(A^{-1}\right)^{\prime}\left(A^{i}(0)\right)\right)^{1 / 2}
$$

and

$$
A^{-1}(x)+n-1=A^{-1}(x)-(1-n)=x\left(\left(A^{-1}\right)^{\prime}(x) \cdot\left(A^{-1}\right)^{\prime}(A(1-n))\right)^{1 / 2} .
$$

Therefore

$$
\mathcal{U}=\frac{n-1+A^{i-1}(0)}{\left[\left(x-A^{i}(0)\right) \sqrt{\left(A^{-1}\right)^{\prime}\left(A^{i}(0)\right)}\right]\left[x \sqrt{\left(A^{-1}\right)^{\prime}(A(1-n))}\right]} .
$$

Another application of Lemma 1 gives

$$
A^{i}(0)=A\left(A^{i-1}(0)\right)-A(1-n)=\left(n-1+A^{i-1}(0)\right) \sqrt{A^{\prime}(1-n) \cdot A^{\prime}\left(A^{i-1}(0)\right)} .
$$

Using $\left(A^{-1}\right)^{\prime}\left(A^{i}(0)\right) A^{\prime}\left(A^{i-1}(0)\right)=1$ and $\left(A^{-1}\right)^{\prime}(A(1-n)) A^{\prime}(1-n)=1$, we conclude that

$$
\begin{gathered}
\mathcal{U}=\frac{A^{i}(0)}{\left(x-A^{i}(0)\right) \cdot x \cdot \sqrt{\left(A^{-1}\right)^{\prime}\left(A^{i}(0)\right) A^{\prime}\left(A^{i-1}(0)\right)} \cdot \sqrt{\left(A^{-1}\right)^{\prime}(A(1-n)) A^{\prime}(1-n)}} \\
=\frac{A^{i}(0)}{x\left(x-A^{i}(0)\right)}=\frac{1}{x-A^{i}(0)}-\frac{1}{x} .
\end{gathered}
$$


The $j$-th term of $\mathcal{V}$ can be obtained from $\mathcal{U}$ by replacing $x$ by $x+j$ and $i$ by $k$. Thus we obtain from the formula for $\mathcal{U}$ the following formula for $\mathcal{V}$.

$\mathcal{V}=\sum_{j=1}^{n-1}\left(\frac{1}{x+j-A^{k}(0)}-\frac{1}{x+j}\right)=\sum_{j=1}^{n-1}\left(\frac{1}{x+j-1}-\frac{1}{x+j}\right)=\frac{1}{x}-\frac{1}{x+n-1}$

Hence

$$
\mathcal{U}+\mathcal{V}=\frac{1}{x-A^{i}(0)}-\frac{1}{x+n-1}
$$

as required. The computation of $\mathcal{V}$ also gives the result when $x \in[0, A(0))$.

Theorem 2. $T$ is ergodic with respect to Lebesgue measure.

Lemma 2. Let $C(x)=(a x+b) /(c x+d)$ be a real Möbius transformation with imaginary fixed points and $a, b>0$. Then for $x \in D=\left(-\infty, C^{-1}(\infty)\right), C^{\prime}(x)>1$ if and only if $|x|<C(x)$. Also, for all $x \in D, C^{\prime \prime}(x)>0$.

Proof. Without loss of generality $a d-b c=1$. Since the fixed points of $C$ are complex, $C$ is an elliptic transformation [2] and $(a+d)^{2}<4$. The real part of the fixed points is then $a-d=0$, and therefore $d=a$ and $0 \leq a<1$. For the fixed points $\pm \sqrt{b / c}$ to be imaginary, $c$ must be negative.

$C$ is defined everywhere on $D=(-\infty,-a / c)$ and since $C^{\prime}(x)=1 /(c x+a)^{2}>0$, it is an increasing function. $C^{\prime}(x)=1$ has the two solutions $x_{1}=(-a+1) / c$ and $x_{2}=-(a+1) / c$, with $x_{1}<0<x_{2}$. Since $x<-a / c$ and $c<0$ implies that $c x+a>0$ for all $x \in D$, it follows that $C^{\prime \prime}(x)>0$ on $D$. Therefore $C^{\prime}(x)>1$ for $x \in D$ if and only if $x \in\left(x_{1}, x_{2}\right) \cap D=\left(\frac{-a+1}{c}, \frac{-a}{c}\right)$.

Then for $x \in D$ non-negative, both $C^{\prime}(x)>1$ and $x<C(x)$, proving the lemma for $x \geq 0$. Suppose $x \in D$ is negative. Consequently, $-x \leq C(x)$ if and only if $0 \leq c x^{2}+2 a x+b$, if and only if $x \in[(-a+1) / c, 0)$, if and only if $C^{\prime}(x)>1$.

The proof of Theorem 2 is based on Thaler's treatment of a large class of maps with infinite invariant measures [6]. In order for those results to be applicable, it is necessary to define a new map induced by $T$ for which the indifferent periodic orbit becomes a fixed point. Let $E_{\ell}=\left(A^{\ell-1}(0), A^{\ell}(0)\right)$ for $1 \leq \ell \leq k$, and define the transformation $S:[0,1] \rightarrow[0,1]$ by $S(1)=1$ and

$$
S(x)=A^{k+1-\ell} \text { for } x \in E_{\ell} .
$$

As an application of Lemma 2 we have:

Corollary 1. For $x \in E_{\ell}, S^{\prime}(x)=\left(A^{k+1-\ell}\right)^{\prime}(x) \geq 1$ with equality if and only if $\ell=1$ and $x=0$. Also, $\left(A^{k+1-\ell}\right)^{\prime \prime}(x)>0$ for $x \in E_{\ell}$.

Proof. It follows from $A^{k+1-\ell}\left(E_{\ell}\right)=\left[A^{k}(0), A^{k+1}(0)\right)=[1, n)$ that

$$
E_{\ell} \subseteq\left(-\infty, A^{\ell-k-1}(\infty)\right)
$$

Let $f(x)=x+1 / 2$ and consider the transformation $C(x)=f^{-1} \circ A^{k+1-\ell} \circ f(x)$. First note that $C^{\prime}(x)=\left(A^{k+1-\ell}\right)^{\prime}(f(x))$ and $f^{-1}\left(E_{\ell}\right) \subseteq D$ where $D$ is the domain defined in Lemma 2 with respect to $C$. The previous lemma is applicable and we infer that $C^{\prime}(x) \geq 1$ on $f^{-1}\left(E_{\ell}\right)$ if and only if $|x| \leq C(x)$. In terms of $A^{k+1-\ell}$ that says $\left(A^{k+1-\ell}\right)^{\prime}(x) \geq 1$ on $E_{\ell}$ if and only if $|x-1 / 2| \leq A^{k+1-\ell}(x)-1 / 2$. Since $x \in[0,1)$ and $A^{k+1-\ell}(x) \geq 1$, it follows that $\left(A^{k+1-\ell}\right)^{\prime}(x) \geq 1$. The only chance 
for equality occurs when $x=0$ and $A^{k+1-\ell}(x)=1$, that is, exactly when $l=1$. The last statement of the corollary follows from the last part of Lemma 2.

Proof of Theorem 2. By (1.1) in [6], if $S$ is shown to be ergodic, then the theorem will follow. Further refine the covering by defining $B_{\ell j}=S^{-1}([j, j+1)) \cap E_{\ell}$ for $j=1, \ldots, n-1$ and modifying $B_{k, n-1}$ by including the endpoint 1 . Set $I=$ $\{(\ell, j) \mid 1 \leq \ell \leq k, 1 \leq j \leq n-1\}$. Then $\bigcup_{i \in I} B_{i}=[0,1]$ and for each $i \in I,\left.S\right|_{B_{i}}$ is a homeomorphism of $B_{i}$ onto $[0,1)$ (or $[0,1]$ ) which extends to a homeomorphism of $\overline{B_{i}}$ onto $[0,1]$.

To show that $S$ is ergodic, in fact exact, we verify the hypotheses of [6], Theorem 1. The hypotheses are similar to those that are encountered in the "folk theorem" for expanding Markoff maps of the interval ([1] and [3]), with some additions that deal with the indifferent fixed points. $S$ extends to a Möbius transformation on $\overline{B_{i}}$ so that $\left.S\right|_{\overline{B_{i}}}$ is $C^{\infty}$ for each $i \in I$. Therefore both $\left|S^{\prime \prime}(x)\right|$ and $0<S^{\prime}(x)$ are bounded on $[0,1]$ and consequently, $\left|S^{\prime \prime}(x)\right| S^{\prime}(x)^{-2}$ is bounded on $[0,1]$. From the corollary we have $S^{\prime}(x) \geq 1$ with equality if and only if $x=0$. By the intermediate value theorem the extended $\left.S\right|_{\overline{B_{i}}}$ has a fixed point on each $\overline{B_{i}}$, and since $S^{\prime}(x)>1$ except at zero, the fixed point is unique. Again, from the corollary $S^{\prime \prime}(x)>0$ for $x \in[0,1)$ and in particular, $S^{\prime}$ is increasing on the interval $B_{11}$ which contains zero. Finally, for each $i \in I, \overline{S\left(B_{i}\right)}=[0,1]$.

Example 1. When $k=2, \alpha=1 / 2$ independent of the value $n$. Then $A(x)=$ $(-x-n+1) /((2 n+3) x+2-2 n)$ and the invariant density takes the form

$$
\rho(x)=\chi_{[0,1 / 2)}(x) \frac{1}{x}+\chi_{[1 / 2,1)}(x) \frac{1}{x-1 / 2}-\frac{1}{x+n-1} .
$$

\section{REFERENCES}

[1] R. L. Adler. Geodesic flows, interval maps and symbolic dynamics. In "Ergodic Theory, Symbolic Dynamics and Hyperbolic Spaces", T. Bedford, H. Keane, C. Series, eds., Oxford Univ. Press, 1991. CMP 92:02

[2] A. F. Beardon, "The Geometry of Discrete Groups" Graduate Texts in Math. 91, SpringerVerlag, Berlin-Heidelberg-New York, 1987. MR 97d:22011

[3] I. P, Cornfeld, S.V. Fomin and Ya. G. Sinai. Ergodic Theory, Springer-Verlag, BerlinHeidelberg-New York, 1982. MR 87f:28019

[4] K. Gröchenig and A. Haas, Backward continued fractions, invariant measures, and mappings of the interval. Ergodic Th. and Dyn. Sys. 16 (1996), 1241-1274. MR 97m:58114

[5] W. de Melo and S. van Strien, One-Dimensional Dynamics, Ergebnisse d. Math. 25, SpringerVerlag, Berlin-Heidelberg-New York, 1993. MR 95a:58035

[6] M. Thaler, Transformations on $[0,1]$ with infinite invariant measure. Israel J. Math. 46 (1983), 67-96. MR 85g:28020

Department of Mathematics U-3009, The University of Connecticut, Storrs, ConNECTICUT 06269-3009

E-mail address: groch@math.uconn.edu

E-mail address: haas@math.uconn.edu 